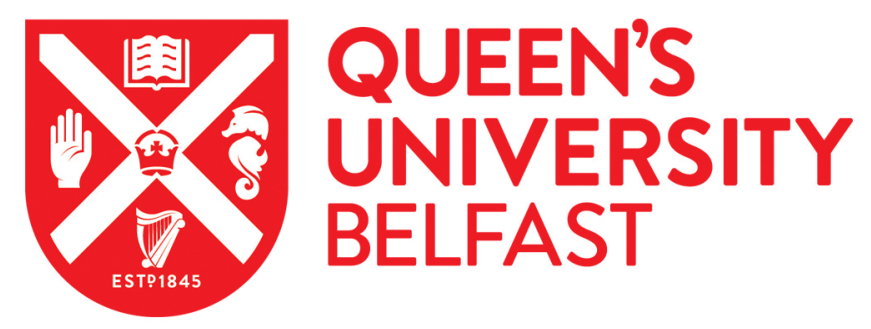

\title{
Expanding the portfolio of tribo-positive materials: aniline formaldehyde condensates for high charge density triboelectric
} nanogenerators

Zhao, P., Soin, N., Kumar, A., Shi, L., Guan, S., Tsonos, C., Yu, Z., Ray, S. C., McLaughlin, J., Zhu, Z., Zhou, E., Geng, J., See, C. H., \& Luo, J. (2020). Expanding the portfolio of tribo-positive materials: aniline formaldehyde condensates for high charge density triboelectric nanogenerators. Nano Energy, 67(104291), [104291]. https://doi.org/10.1016/j.nanoen.2019.104291

Published in:
Nano Energy

Document Version:

Peer reviewed version

Queen's University Belfast - Research Portal:

Link to publication record in Queen's University Belfast Research Portal

\section{Publisher rights}

Copyright 2019 Elsevier.

This manuscript is distributed under a Creative Commons Attribution-NonCommercial-NoDerivs License

(https://creativecommons.org/licenses/by-nc-nd/4.0/), which permits distribution and reproduction for non-commercial purposes, provided the author and source are cited.

\section{General rights}

Copyright for the publications made accessible via the Queen's University Belfast Research Portal is retained by the author(s) and / or other copyright owners and it is a condition of accessing these publications that users recognise and abide by the legal requirements associated with these rights.

\section{Take down policy}

The Research Portal is Queen's institutional repository that provides access to Queen's research output. Every effort has been made to ensure that content in the Research Portal does not infringe any person's rights, or applicable UK laws. If you discover content in the

Research Portal that you believe breaches copyright or violates any law, please contact openaccess@qub.ac.uk. 


\section{Expanding the portfolio of tribo-positive materials: aniline formaldehyde condensates for high charge density triboelectric nanogenerators}

Pengfei Zhao, ${ }^{1}$ Navneet Soin, ${ }^{2,}{ }^{*}$ Amit Kumar, ${ }^{3}$ Lin Shi, ${ }^{4}$ Shaoliang Guan, ${ }^{5,6}$ Christos Tsonos, ${ }^{7}$ Zidong Yu, ${ }^{1}$ Sekhar Chandra Ray, ${ }^{8}$ James A. McLaughlin, ${ }^{2}$ Zhigang Zhu, ${ }^{9}$ Erping Zhou, ${ }^{1}$ Junfeng Geng, ${ }^{1}$ Chan H. See, ${ }^{10}$ and Jikui Luo ${ }^{1}$

${ }^{1}$ Institute for Materials Research and Innovation (IMRI), School of Engineering, University of Bolton, Bolton BL3 5AB, United Kingdom

${ }^{2}$ School of Engineering, Ulster University, Newtownabbey, Belfast BT37 0QB, Northern Ireland, United Kingdom

${ }^{3}$ School of Mathematics and Physics, Queen's University Belfast, University Road, Belfast BT7 1NN, Northern Ireland, United Kingdom

${ }^{4}$ College of Information Science and Electronic Engineering, Zhejiang University, 38 Zheda Road, Hangzhou 310027, P. R. China

${ }^{5}$ HarwellXPS, Research Complex at Harwell (RCaH), Didcot, OX11 0FA, United Kingdom.

${ }^{6}$ School of Chemistry, Cardiff University, Cardiff, CF10 3AT, United Kingdom.

${ }^{7}$ Department of Physics, University of Thessaly, $3^{\text {rd }}$ Old National Road, Lamia-Athens, 35100 Lamia, Greece

${ }^{8}$ Department of Physics, College of Science, Engineering and Technology, University of South Africa, Private Bag X6, Florida, 1710, Johannesburg, South Africa

${ }^{9}$ School of Environmental and Materials Engineering, College of Engineering, Shanghai Polytechnic University, 201209, Shanghai, P. R. China

${ }^{10}$ School of Engineering \& the Built Environment, Edinburgh Napier University, Edinburgh, EH1 1 4BN, United Kingdom

*Corresponding author email: $\underline{\text { n.soin@,ulster.ac.uk }}$ 


\section{Abstract}

The rapid uptake of energy harvesting triboelectric nanogenerators (TENGs) for self-powered electronics requires the development of high-performance tribo-materials capable of providing large power outputs. This work reports on the synthesis and use of aniline formaldehyde resin (AFR) for energy-harvesting applications. The facile, acidic-medium reaction between aniline and formaldehyde produces the aniline-formaldehyde condensate, which upon an in-vacuo high temperature curing step provides smooth AFR films with abundant positively-charged nitrogen and oxygen surface functional groups, endowing AFR with a significantly higher positive tribo-polarity than the existing state-of-art polyamide-6 (PA6). A TENG comprising of optimized thin-layered AFR against a polytetrafluoroethylene (PTFE) film produced a peakto-peak voltage of up to $\sim 1,000 \mathrm{~V}$, a current density of $\sim 65 \mathrm{~mA} \mathrm{~m}^{-2}$, a transferred charge density of $\sim 200 \mu \mathrm{C} \mathrm{m}^{-2}$ and an instantaneous power output (energy pulse) of $\sim 11 \mathrm{~W} \mathrm{~m}^{-2}\left(28.1 \mu \mathrm{J}\right.$ cycle ${ }^{-}$ ${ }^{1}$ ), respectively. The suitability of AFR was further supported through the scanning Kelvin probe force microscopy (SKPFM) measurements, which reveal a significantly higher surface potential value of $1.147 \mathrm{~V}$ for AFR as compared to $0.87 \mathrm{~V}$ for PA6 and a step-by-step increase of the surface potential with the increase of energy generation cycles. The work not only proposes a novel mouldable AFR synthesis process but also expands with excellent prospects, the current portfolio of tribo-positive materials for triboelectric energy harvesting applications.

Keywords: Aniline formaldehyde resins; tribo-positive polymers; triboelectric nanogenerators; surface potential; KPFM 


\section{Introduction}

It is well established that the contact charging/electrification phenomena generates an equal and opposite amount of surface charges when two material surfaces are brought into contact, with charges being injected from one material to another. This interfacial charge flow was first empirically established in 1757 by Wilcke [1], and since then materials have been arranged in a specific triboelectric series by Henniker [2] and more recently by the Wang group [3], according to their ability to lose or gain interfacial charge species, thus, classifying them as tribo-positive or tribo-negative materials, respectively. In the case of dielectric materials, these exchanged charges remain on the surface, leading to the formation of uncompensated dipoles, which can be extracted using the metal electrodes as the harvested charge [3]. Indeed, this phenomenon has been used to develop triboelectric nanogenerators (TENGs), which have been utilized to convert various forms of ambient mechanical energy to electrical energy [3-8]. The commonly reported vertical contact mode TENGs typically consist of a combination of dielectric/dielectric or dielectric/metal, with significant differences in their ability to donate (tribo-positive) or accept electrons (tribo-negative). For these TENG systems, the increase in the output power and surface charge density is achieved via: (i) judicious selection of tribomaterials [8], (ii) surface micro-/nano-structuring [9,10], surface chemistry modification [11], (iii) charge injection [6] or composites with high-polarization piezoelectric additives $[5,7,12]$ and (iv) physical hybridization of multiple energy harvesting technologies [13]. Except for the judicious use of tribo-materials, the rest of the methods are reliant on expensive equipment and complicated processing steps including lithography etc. which defeats the low-cost advantage of TENGs and may not provide the required stability and reliability. Consequently, the selection of the optimal materials with high electron affinity difference is the simplest and most effective way to improve the performance of TENGs but is currently restricted by the limited choices of existing materials.

With excellent dielectric properties, mechanical robustness and ease of fabrication, both natural and synthetic polymers feature extensively in the triboelectric series and display consistent charge transfer patterns corresponding to specific surface chemical moieties. A preliminary inspection of the chemical structure of these polymers reveals the following material design principles: (i) nitrogen-containing polymers with pyridine, amine and amide groups (e.g. nylon, polyvinyl-2-pyridine), develop the most positive charge $\left(0.5-1.2 \mathrm{pC} \mathrm{cm}^{-2}\right)$, which is slightly higher than the polymers with oxygen functional (e.g. polyethylene oxide, polyvinyl alcohol)

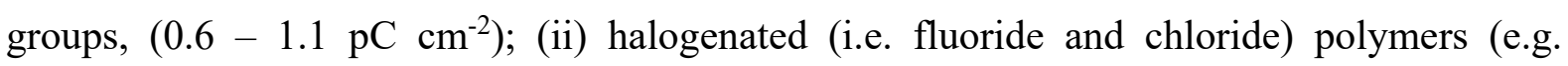


polyvinylchloride, polytetrafluoroethylene (PTFE)) develop the most negative charge (-1.6 - $2.8 \mathrm{pC} \mathrm{cm}^{-2}$ ); and (iii) the hydrocarbons contribute almost nothing to the tribo-polarity[14-16]. In the first ever report of an empirical triboelectric series by Henniker, it was reported that the series itself showed no correlation with the dielectric constant or the dipole moment but was dependent on a determinant property linked to molecular constitution especially that of the surface [2]. While it is relatively more easy to associate the halide groups (-I, - $\mathrm{Br}$ and $-\mathrm{F})$ with the tribo-negative behaviour owing to their high electronegativity; the tribo-positive behaving polymers were observed to be hydrophilic, with high dielectric constants and highly polar with nitrogen and oxygen surface functional groups [17]. In their report, Ohara et al. [18], have reported on the triboelectrification of Langmuir-Blodgett films wherein various polar groups $\left(-\mathrm{COOH},-\mathrm{CONH}_{2},-\mathrm{CONHC}_{6} \mathrm{H}_{5},-\mathrm{COOCH}_{3},-\mathrm{CH}_{2} \mathrm{OH}\right.$ and $\left.-\mathrm{CH}_{2} \mathrm{NH}_{2}\right)$ were bonded to the same type of hydrocarbon chains $\left(\mathrm{CH}_{3}\left(\mathrm{CH}_{2}\right)_{16-}\right)$. It was observed that the electrification between the monolayers and glass by taking the polarity and the magnitude was as following: $(+)-\mathrm{CH}_{2} \mathrm{NH}_{2}$, $-\mathrm{CH}_{2} \mathrm{OH},-\mathrm{COOH},-\mathrm{CONHC}_{6} \mathrm{H}_{5},-\mathrm{CONH}_{2},-\mathrm{COOCH}_{3}$ (-) with the local dipole moments of the atomic groups being $0.30,0.26,0.01,-0.20,0.17$ and $0.34 \mathrm{D}$, respectively. From their data, it seems that there is a direct correlation between the magnitude of the dipole moment with the atomic groups and their corresponding electron density [18]. Similarly, in their work, Shinohara et al reported that for the triboelectric series incorporating the p-substituted styrene polymers, the modified polymers with electron-releasing groups are situated on the positive side, while the electron-attracting polymers are located on the negative side [19]. More importantly, in the case of polymers with nitrogen atoms in polar groups, those with a high electron density on the nitrogen atoms are charged positively, while those with a low electron density were charged negatively [19]. For instance, polymers with an amino group such as poly(p-dimethylaminostyrene) and poly(p-aminostyrene) and those possessing a carbonyl group such as poly- (methylmethacrylate) and poly(vinyl acetate) charged positively when measured against a metal. The presence of lone pair of electrons on the nitrogen atom (in amino groups) was considered to affect the triboelectrification greatly and corroborates the fact that the electronic and chemical surface state of polymers greatly affects the tribolectric behaviour [19]. Thus, the ranking for polymers tends to be determined by their inherent chemical bonding, which affects the molecular constitution of the contact interface and hence the interfacial charge transfer. As polyvinylidene fluoride (PVDF) and PTFE are among the best performing tribo-negative materials, only a few improvements have been brought to these materials such as fluorinated polydimethylsiloxane [20], plasma-treated PTFE [21] and fluorinated ethylene propylene [22]. However, not much attention has been paid to the selection of tribo-positive 
materials, which are equally crucial for enhancing the surface charge density and subsequent power density of TENGs. In our previous work, we had introduced the use of flat-surfaced PEO as a tribo-positive material which is capable of providing a high surface charge density of nearly $160 \mu \mathrm{C} \mathrm{m}^{-2}$ against PDMS [8]. In comparison, the PA6/PDMS based TENGs showed a charge density of $110 \mu \mathrm{C} \mathrm{m}^{-2}$ only, therefore establishing the superiority of PEO over PA6. However, there is a need to expand the range of available tribo-positive materials further.

Henniker et al. have reported that condensation products of aniline formaldehyde reactions with abundant amide groups acquire the most amount of positive contact charge [2]. The aniline-formaldehyde condensates are thermo-reactive products of chemical interaction between aniline hydrochloride and formaldehyde in an acidic solution and can be synthesized as linear or cross-linked condensation polymers by varying the molar ratio of aniline to formaldehyde during polymerization [20-23]. The cured aniline formaldehyde resins (AFR) have shown to be an effective inhibitor of corrosion on mild steel in acidic medium by forming a protective layer of $-\mathrm{C}=\mathrm{N}$ - on the metal surface [24,25]. Similarly, by adding an excess of epichlorohydrin, a new type of epoxy was synthesized with applications in casting, adhesives and encapsulation of semiconductor devices [26,27]. The cured AFR resins being chemically resistant to alkalis have been widely used for electro-technical plastics and as components for radio- and high-frequency electrical engineering owing to their excellent electrical insulation properties which outperform the melamine-, urea- and phenol-formaldehyde based resins $[20,21]$. However, their use has become quite restricted owing to the difficult moulding process and have fallen out in favour of melamine-type resins. Also, the room-/high-temperature synthesis leads to fast reaction kinetics causing the resinous product to have a random porous structure and with the fast-thermosetting properties, the application scenarios of pristine AFRs have been quite limited.

Herein, we report on the use of AFR films as tribo-positive material to fabricate highperformance TENGs in combination with PTFE. Relying on a facile low-temperature $\left(\sim 0^{\circ} \mathrm{C}\right)$, acidic-medium condensation polymerization of aniline with formaldehyde, the condensate is fully curable at $\sim 130^{\circ} \mathrm{C}$ and can be formed into the required shape with sufficient mechanical rigidity. We demonstrate AFR/PTFE TENG with a peak-to-peak voltage of $\sim 1,000 \mathrm{~V}$, a shortcircuit current density of $65 \mathrm{~mA} \mathrm{~m}^{-2}$ (corresponding a charge density of $\sim 200 \mu \mathrm{C} \mathrm{m}^{-2}$ ), and energy pulse of $\sim 28.1 \mu \mathrm{J} \mathrm{cycle}^{-1}$ with an instantaneous power density of $\sim 11 \mathrm{~W} \mathrm{~m}^{-2}$, all of which are near twice the values reported for PA6/PTFE TENGs. The synthesized AFR is mechanically stable, and the AFR/PTFE TENG shows excellent stability with less than $\pm 5 \%$ 
variation in its electrical output over 16,000 contact cycles. In-depth characterization of AFR samples via thermal, dielectric, nuclear magnetic resonance and X-ray spectroscopic techniques in conjunction with scanning Kelvin probe force microscopy (SKPFM) surface potential measurements supports the suitability of AFR as a high-performance tribo-positive material. The SKPFM measurements show clearly the significantly higher surface potential value of $1.147 \mathrm{~V}$ for AFR as compared to $0.87 \mathrm{~V}$ for PA6, thereby expanding the rather small portfolio of high-performance tribo-positive materials for energy-harvesting applications.

\section{Experimental}

\subsection{Materials and characterization}

Aniline (>99.5\%), hydrochloric acid (37\%) and aqueous formaldehyde solution (37 wt \%, containing 10-15\% methanol as a stabilizer) were purchased from Sigma Aldrich (United Kingdom) and used as such without any further purification. PTFE films ( $\sim 0.03 \mathrm{~mm}$ thickness) for fabricating tribo-negative layers were supplied from the College Hardware, China. The charge injection on PTFE films was carried out using a commercial antistatic gun (Zerostat 3, Milty). Further details on the charge injection process can be found in our earlier work [6].

The curing temperature and thermal behaviour of the synthesized AFR materials were investigated by Differential scanning calorimetry (DSC) system on a TA Instruments DSC Q2000. The samples, approximate weight of $2 \mathrm{mg}$, were heated at $10^{\circ} \mathrm{C} \min ^{-1}$ from $0-200{ }^{\circ} \mathrm{C}$ under a constant $50 \mathrm{~mL} \mathrm{~min}^{-1} \mathrm{~N}_{2}$ flow. Vendor provided software was used to assess parameters of glass transition temperature $\left(\mathrm{T}_{\mathrm{g}}\right)$ and onset, maximum curing temperatures. A Hitachi S3400N scanning electron microscope (SEM) was used to study the surface morphology and cross-section of the AFR and PTFE layers. The Fourier transform infra-red (FTIR) spectra of samples were obtained on a Thermo Scientific IS10 Nicolet at a nominal resolution of $\pm 1 \mathrm{~cm}^{-}$ ${ }^{1}$ for a total of 128 scans and processed through OMINIC. X-ray photoelectron spectroscopy (XPS) was performed on a Thermo Fisher Scientific NEXSA spectrometer using a microfocused monochromatic Al x-ray source $(19.2 \mathrm{~W})$ over an area of approximately 100 microns. Data were recorded at pass energies of $150 \mathrm{eV}$ for survey scans and $40 \mathrm{eV}$ for highresolution scans with $1 \mathrm{eV}$ and $0.1 \mathrm{eV}$ step sizes, respectively. Charge neutralization of the sample was achieved using a combination of both $\mathrm{Ar}^{+}$ions and low energy electrons. Depth profiling was accomplished using the instrument's EX06 monatomic ion source operated at 1 
$\mathrm{keV}$ and rastered over a $3 \times 3 \mathrm{~mm}^{2}$ area at the rate of $0.4 \mathrm{~nm} \mathrm{~s}^{-1}$ (reference $\mathrm{Ta}_{2} \mathrm{O}_{5}$ ) for $2500 \mathrm{~s} .{ }^{1} \mathrm{H}$ Nuclear Magnetic Resonance (NMR) spectra of the cured AFR was obtained in a deuterated dimethyl sulfoxide (DMSO) using a Bruker NMR Spectrometer AV3 HD 500 operated at 600 $\mathrm{MHz}$ and processed with a third-party analytical chemistry software. Dielectric measurements were carried out using Dielectric Relaxation Spectroscopy (DRS) technique employing an Alpha analyser supplied by Novocontrol. For this, an AFR sample of $\sim 1 \mathrm{~mm}$ thickness was placed between two brass electrodes of $20 \mathrm{~mm}$ diameter and inserted as a capacitor in the Novocontrol sample cell. The complex dielectric permittivity was recorded and measured in a broad frequency ranging from 1 to $10^{6} \mathrm{~Hz}$. Nanoscale surface potential mapping was undertaken using an Asylum Research MFP-Infinity atomic force microscope in the SKPFM mode, which allows measurement of true surface potential through operation in a two-pass mode. For SKPFM measurements, Pt-coated Si tips (Nanosensors PPP-EFM) with stiffness constant of $2.8 \mathrm{~N} \mathrm{~m}^{-1}$ were used.

\subsection{Synthesis of AFR}

An acidic medium (20 ml, $10 \mathrm{M} \mathrm{HCl}$ solution) was first prepared by adding $10 \mathrm{ml}$ deionized water to $10 \mathrm{ml}$ of stock $20 \mathrm{M} \mathrm{HCl}$, and further mixed with $15 \mathrm{ml}$ of aniline. The prepared paleyellow coloured solution was then cooled down to $\sim 0{ }^{\circ} \mathrm{C}$ in an ice bath under constant stirring. Subsequently, $7 \mathrm{ml}$ of formaldehyde was added dropwise slowly into the solution under continuous vigorous stirring, during which the solution gradually became a yellow resinous condensate. Owing to the high viscosity of the condensate, the magnetic stirring bar was removed, and further vigorous stirring carried out manually using a glass rod to ensure a complete reaction. The collected condensate was subsequently washed with copious amounts of distilled water to remove the excess unreacted reactants and by-products.

\subsection{Preparation and electrical characterization of TENGs}

Conductive aluminium layers as electrodes were attached to two separate glass slides $(2 \times 2 \times$ $0.1 \mathrm{~cm})$. The tribo-negative layer was produced by attaching a PTFE thin film, while the AFR acted as the opposite layer. For the tribo-positive layer, the obtained gelatinous AFR condensate was spread out evenly on the surface of the prepared Al electrodes (Fig. 1b(i)), washed with distilled water (Fig. 1b(ii)) and further dried overnight in a desiccator (Fig. 1b(iii)). The samples were then cured under optimal conditions (detailed discussion in the following section) to obtain smooth AFR films on Al substrate (Fig. 1b(iv)). The two layers were assembled to form contact mode TENG devices. 
A computer-controlled dynamic fatigue tester system (Popwil Model YPS-1) was used to characterize the TENGs by controlling the contact force $(1-100 \mathrm{~N})$, frequency $(0-10 \mathrm{~Hz})$ and spacer distance of the two tribo-contact layers. An oscilloscope (Tektronix MDO3022) and a picometer (Keysight B2981A) were connected in parallel or serial, respectively, to test the voltage output and the short-circuit current, respectively. The corresponding charge density was derived from the area under the curve of the measured current density. For all the TENG electrical measurements on picometer and oscilloscope, the Al electrode corresponding to the AFR side was connected to the positive lead, while the PTFE side Al was grounded.

\section{Results and discussion}

\subsection{Material characterization}

It is well established that, to achieve maximum cross-linking and ensuing mechanical strength, resins should be cured at the lowest curing temperatures for a longer duration [28]. The aniline formaldehyde condensates are thermosetting resins whose optimal curing conditions were determined using a two-cycle DSC thermal analysis [23]. The thermogram shown in Fig. 1(a) exhibits classic thermoset behaviour. In the first heating run, an exothermic peak was observed at $\sim 120^{\circ} \mathrm{C}$, corresponding to the curing temperature range, while in the second heating run, no further melting/curing peaks were observed except for a glass transition $\left(\mathrm{T}_{\mathrm{g}}\right.$ ) peak at $\sim 31{ }^{\circ} \mathrm{C}$. The second DSC run is essential to assess that the samples are fully cured (indicated by the absence of any further curing exotherm) or whether further post-curing was required [23,28]. Correspondingly, the curing process of the AFR resins on $\mathrm{Al}$ substrates was performed in a vacuum oven $\left(\sim 120^{\circ} \mathrm{C}, \sim 14.5 \mathrm{Psi}\right)$ for 2 hours to eliminate any residual unreacted aniline, formaldehyde and/or other volatiles entirely with no further post-curing. The curing was performed by turning the AFR deposited side upside down onto a flat PTFE substrate to make flat thin films for TENGs. To obtain AFR samples with varying thicknesses, four different weights $(1000 \mathrm{~g} / 500 \mathrm{~g} / 300 \mathrm{~g} / 200 \mathrm{~g})$ were added on to the backside. Subsequently, the oven was switched off, and samples were allowed to cool down to room temperature to obtain the hotpressed AFR thin films on the Al electrode (Fig. 1e (iv)). As observed in the SEM images of Fig. 1(a-d), a significant variation in the surface morphology was observed which can be attributed to the following factors: in-vacuo removal of pores and residual moisture content under heating and the simultaneous application of weight to obtain a smooth free-standing film under vacuum conditions. As the AFR condensate is put through its curing cycle, it softens up 
as it goes through its $T_{g}$ wherein not only the pores are removed due to the vacuum conditions, its viscosity too would reduce allowing it to flow while the simultaneous application of various loads against a smooth PTFE surface allow it to form a continuous fully cured polymeric network devoid of any pores.

The cured AFR sample was characterized by ${ }^{1} \mathrm{H}$ NMR and data shown in Fig. 2(c) with the sharp sextet at $\delta 2.51 \mathrm{ppm}$ being ascribed to the solvent DMSO. Previous work by Zhang et al. has shown that a reduction in the aniline to formaldehyde ratio in the polymerization reaction has a significant effect on the intensity, area and shape of the peak at $\delta 5.10 \mathrm{ppm}$ with corresponding changes in the peaks for aromatic rings and methylene groups [25]. The further multiple peaks appear in the range of $\delta 6.5-7.0 \mathrm{ppm}$, corresponding to the aromatic rings and amino groups; meanwhile, the peaks in the range of $\delta 3.55-3.67 \mathrm{ppm}$ correspond to the methylene group $\left(-\mathrm{CH}_{2}-\right)$ groups connecting the aromatic rings $[22,25,29]$. The broad peak around $\delta 5.50 \mathrm{ppm}$ corresponds to the primary and secondary amine protons $[22,25,29]$. By integration of the ${ }^{1} \mathrm{H}$ NMR signals, the relative molecular ratio of the prepared for aromatic rings, methylene and amino groups were calculated to be 10:4:5 which is similar to those reported by Zhang et al. [25].

The molecular structure of the AFR samples before and after curing was confirmed with FTIR spectroscopy. By observing Fig. 2(d), the bands at $\sim 1,515 \mathrm{~cm}^{-1}$ and $\sim 812 \mathrm{~cm}^{-1}$ can be assigned to the stretching vibration of the benzenoid framework and the bending vibration of the substituted benzene ring [22,25]. The absorption bands range from 1,630-1,500 $\mathrm{cm}^{-1}$, and $1,350-1,250 \mathrm{~cm}^{-1}$ are assigned to $-\mathrm{NH}$ and $-\mathrm{C}-\mathrm{N}$ - vibrations and stretching, respectively; while the bands in the range of $3,500-3,300 \mathrm{~cm}^{-1}$ are due to the primary and/or secondary amine functional groups $[22,25,30]$. The peaks in the range of $1,400-1,300 \mathrm{~cm}^{-1}$ indicate the existence of $-\mathrm{CH}_{2}-\mathrm{N}$ bonding. Stretching vibrations of aromatic protons result with peaks at $\sim 2,900 \mathrm{~cm}^{-}$ ${ }^{1}$ and out of plane bending vibrations in the range of 780-650 $\mathrm{cm}^{-1}$ [31]. After the curing process, the intensities decrease for the absorption bands at $\sim 3,340 \mathrm{~cm}^{-1}$ and $\sim 1,619 \mathrm{~cm}^{-1}$, indicating the presence of lower primary and secondary amine units in the molecular structure, which could lead to the formation of the final product with much lower solubility [22]. Although it is generally recognized that polymer chemical structure cannot be fully identified from the spectral results only, previous work by Zhang et al.[25] have shown that a linear copolymer is formed when an excess of aniline is used (similar to the one used in our work), in which the amino group of aniline unit predominantly exists as primary and/or secondary amine. As mentioned earlier in the experiment section, the yellow residual solution indicates an excess 
amount of aniline and the presence of primary and secondary amines can be confirmed by the existence of strong absorption bands at $\sim 1,600 \mathrm{~cm}^{-1}$ and $\sim 1,268 \mathrm{~cm}^{-1}$ of the FTIR results [22,25], as observed in our samples. The probable reaction mechanism and structures of the synthesized products are proposed by combining all the obtained spectra results, which is shown in Fig. $3[20]$.

Fig. 2(c) shows the core-level XPS spectra for the carbon, nitrogen and oxygen, while quantification using appropriate relative sensitivity factors reveals the surface composition, carbon (81.5 at.\%), nitrogen (8.9 at.\%) and oxygen (9.6 at.\%). All the high-resolution corelevel spectra were fitted using Gaussian components to identify the diverse chemical bonding states present in the AFR samples. The high binding energy asymmetrical C 1s spectrum was deconvoluted into four components centred at $284.8 \mathrm{eV}, 285.8 \mathrm{eV}, 287.0 \mathrm{eV}$ and $288.7 \mathrm{eV}$, corresponding to $\mathrm{sp}^{2}$ bonds, $\mathrm{C}-\mathrm{N}$ bonds in amino groups and/or amine-sided carbon atoms, $\mathrm{C}-$ $\mathrm{O}$ groups and $\mathrm{C}=\mathrm{O}$ groups, respectively apart from the satellite peak at $\sim 291 \mathrm{eV}$ [32]. The $\mathrm{N}$ 1s spectra show only a single peak at $\sim 399.7 \mathrm{eV}$, corresponding to the nitrogen bonded to carbon in amino and/or amino and/or amide groups [32,33]. This peak has also been attributed to an overlap of the amine, amide and imide contributions for nitrogen-containing polymers $[32,33]$. The $\mathrm{O} 1 \mathrm{~s}$ spectra show the presence of components at $531.5 \mathrm{eV}$ and $532.9 \mathrm{eV}$, corresponding to $\mathrm{C}-\mathrm{O}$ and $\mathrm{C}=\mathrm{O}$ groups, respectively $[32,33]$. It should be noted that oxygen is not usually expected in the chemical structure of AFR, but owing to the low-vacuum curing process, surface oxygen is observed which is absent in the bulk of the material (as confirmed from in-situ XPS Ar ${ }^{+}$sputtering (see Fig. S2, Supporting Information). As discussed earlier, both the nitrogen and oxygen groups can impart tribo-positive functionality to the surface owing to their electron-donating capabilities wherein the nature of the oxygen group (ether, hydroxyl or carbonyl), has only a small effect on the magnitude of the charge. For AFR which has abundant nitrogen and oxygen functional groups, a high tribo-positive behaviour is therefore expected.

For the application of the cured AFR samples in a capacitive type TENG device, it is essential to study their dielectric behaviour. including the variation of the real part $\left(\varepsilon^{\prime}\right)$, and the loss $\operatorname{tangent} \tan \delta\left[\tan \delta=\varepsilon^{\prime \prime} / \varepsilon^{\prime}\right]$ (where $\varepsilon^{\prime \prime}$ presents the imaginary part) of dielectric constant with the variation of frequency. It can be observed (Fig. 2(d)) that the $\varepsilon^{\prime}$ of AFR at room temperature decreases from a value of 3.96 at $1 \mathrm{~Hz}$ to a value of 3.17 at $1 \mathrm{MHz}$, similar to those referred to the literature [34]. When the frequency was higher than $10 \mathrm{~Hz}, \tan \delta$ shows values of less than 0.2 in all the frequency window. Especially for frequencies higher than $1 \mathrm{kHz}, \tan \delta$ presents a 
value lower than 0.01 . The near-linear increase of $\tan \delta$ below $1 \mathrm{kHz}$ may be associated with the low-frequency dielectric relaxation, which is accompanied by a corresponding greater increment of $\varepsilon^{\prime}$ values. This trend of the dielectric parameters matches well with Koop's theory and Maxwell-Wagner model, which describes the interfacial dipoles have less time to orient themselves in the direction of the high-frequency alternating field, thus decreasing the polarization at higher frequencies [35]. However, as the contact-mode TENG is commonly operated in the low-frequency range $(1-10 \mathrm{~Hz})$, the effect of variation of the dielectric constant on the TENG output is minimal (see Eqs. 1 \& 2) [36].

\subsection{Electrical characterization}

The measured output performance of the produced AFR/PTFE TENG is shown in Fig. 4. For the short-circuit condition, the transferred charge for the contact-separate mode TENG devices can be theoretically represented by [36]:

$$
Q_{s c}=\frac{S \sigma x(t)}{d_{0}+x(t)}
$$

Where $S$ is the plane contact area, $\sigma$ is the surface electrification charge density, $x(t)$ is the realtime distance between two contact layers, and $d_{0}$ is the effective thickness represented by:

$$
d_{0}=\frac{d_{1}}{\varepsilon_{1}}+\frac{d_{2}}{\varepsilon_{2}}
$$

Where $\varepsilon_{1}$ and $\varepsilon_{2}$ represent the relative dielectric constants of the two contact triboelectric contact layers of thicknesses $d_{1}$ and $d_{2}$, respectively [36]. Considering the Eq. (1) \& (2), the $d_{1}$ and $\mathrm{d}_{2}$ have a profound effect on the produced voltage output and charge density. To explore this parameter, samples of varying thickness on the $\mathrm{Al}$ electrodes prepared by controlling the load during the in-vacuo hot-press process. With the increase of the pressing load $(200 \mathrm{~g} / 300 \mathrm{~g} / 500 \mathrm{~g} / 1000 \mathrm{~g})$, the thickness of the AFR films reduced from $\sim 973 \mu \mathrm{m}$ to $\sim 117 \mu \mathrm{m}$. As expected, the output voltage and transferred charge density increased with the reduction in the AFR layer thickness (Fig. 4(a\&b)) and the trend was confirmed for all the possible contactforce conditions. For the highest performance, thinner dielectric layers are preferred owing to the better charge induction on the back metal electrodes [36]. Therefore, for further experiments, only samples of $\sim 117 \mu \mathrm{m}$ AFR samples were considered.

Unlike the piezoelectric energy harvesters, which require operation at their resonant frequencies, the TENG devices are high-efficiency mechanical energy to electrical energy 
converters in the low-frequency $(1-10 \mathrm{~Hz})$ region [37,38]. As shown in Fig. 4(c, d) the output voltage and current followed this pattern to give maximum voltage output of $\sim 900 \mathrm{~V}$ and current density of $\sim 60 \mathrm{~mA} \mathrm{~m}^{-2}$ when the contact forces and spacer distance were fixed as $50 \mathrm{~N}$ and $5 \mathrm{~mm}$, respectively, while the short-circuit charge density remained relatively constant at a value of $200 \mu \mathrm{C} \mathrm{m}^{-2}$. However, for the energy harvesting purpose, the transferred charge density is the most crucial output parameters. It can be observed that the maximum transferred charge density value $\left(\sim 225 \mu \mathrm{C} \mathrm{m}^{-2}\right)$ was obtained at the working frequency of $5 \mathrm{~Hz}$, which indicated the optimized working condition of the AFR/PTFE TENG.

According to the literature [36,39] and indeed our previous reports [5-8], the impact force and impact frequency are the two most important operating parameters that affect the contactseparation mode TENGs outputs. However, surprisingly, within the contact force range of 20$80 \mathrm{~N}$, the AFC/PTFE device gave a near-constant level of peak-to-peak voltage output ( $\left.\mathrm{V}_{\mathrm{p}-\mathrm{p}}\right)$ of $\sim 1,000 \mathrm{~V}$ (Fig. 4(c)), albeit at lower contact forces, this output dropped off fairly quickly (see Fig. S3, Supplementary Information). For the current output shown in Fig. 4(d), although the peak values of the current density increase from $\sim 45 \mathrm{~mA} \mathrm{~m}^{-2}$ to $\sim 65 \mathrm{~mA} \mathrm{~m}^{-2}$, the effective transferred charge density stays around $200 \mu \mathrm{C} \mathrm{m}^{-2}$. This relatively constant output, unaffected by the variation of contact force is attributed to the high hardness, flat contact surface and nonvariable polarization properties of the cured AFR substrate. The slight variation of the observed current density may arise due to the malleability of the relatively soft PTFE film, which leads to a variation of film thickness at different impact forces and pressures. It should be noted that this value of $\sim 200 \mu \mathrm{C} \mathrm{m}^{-2}$ is similar to the values reported for the TENGs based on liquid metal gallium $\left(\sim 219 \mu \mathrm{C} \mathrm{m}^{-2}\right)$ [37] but lower than that of soft material and fragmental contacting structure (silicone rubber mixed with nanocarbons) which reach a value of $\sim 250 \mu \mathrm{C} \mathrm{m}^{-2}$ which is considered as the maximum charge density of TENGs working in air environment [38]. Thus, the AFR represents a relatively straightforward material and process for achieving high surface charge density without the need for expensive liquid metals or polymer compounding steps.

The output power of the AFR/PTFE TENG was tested by connecting various external load resistors in series (operating conditions: $50 \mathrm{~N}$ force, $5 \mathrm{~mm}$ spacer distance and $5 \mathrm{~Hz}$ working frequency. The accurate instantaneous peak power values $\left(\mathrm{P}=\mathrm{I}^{2} \times \mathrm{R}\right)$ can be obtained by measuring the current across each resistor. It can be observed in Fig. 5(a) that the output power of AFR-PTFE TENG was maximized at a load resistance of $100 \mathrm{M} \Omega$, corresponding to a peak power density of nearly $11 \mathrm{~W} \mathrm{~m}^{-2}$. However, it has been shown that the instantaneous power output is related to not only the amount of charge transferred, but also to the duration of the 
charge transfer time. Indeed, such an effect has been applied to design micro/nano-switch structure for developing TENG based applications which require high-magnitude voltage peaks [43]. Therefore, instead of the instantaneous peak power, the energy (E) derived from the area under the power curve of each energy generation cycle at the impedance matching condition is much more representative of the output electric energy characterization. This energy, E, is given by:

$$
E=\int_{n t}^{(n+1) t} \mathbf{I}^{2}(t) \cdot \mathbf{R}_{\mathrm{load}} d t
$$

where $I$ stands for the measured current across the load resistance (RLoad), $n$ is the number of generation cycle, and $t$ is the time period of each energy generation cycle $(0.2 \mathrm{~s}$ at $5 \mathrm{~Hz}$ working frequency) [44, 45]. At the impedance matching condition (when $\mathrm{R}_{\mathrm{Load}}=100 \mathrm{M} \Omega$ ), the energy harvested was calculated to be $\sim 28.1 \mu \mathrm{J}_{\text {cycle }^{-1}}$ (Fig. 5(b)), which is nearly twice that that harvested from a PA6/electrospun-PTFE based TENG reported in our earlier work $(\sim 13.8 \mu \mathrm{J}$ cycle $^{-1}$, see Fig S4, Supplementary Information) measured in our previous work [6].

The output stability of the AFR/PTFE TENG was demonstrated by a continuous operation for over 16,000 energy generation cycles at 50 N, $5 \mathrm{~Hz}$ condition). As shown in Fig. 5(c, d), the output variation is within the mechanical system error range $( \pm 5 \%)$ of the contact forces. The continuous stable output demonstrates not only the duration of the tribo-charge on the selected dielectric material surfaces but also the sufficient mechanical strength of the fabricated AFR layer for the long-term energy generation applications. The AFR/PTFE TENG provides enough power to immediately light up 32 chip LEDs without the need for any charge storage (Fig. $5(\mathrm{e}))$.

\subsection{The mechanism for high performance of AFR/PTFE TENGs}

Under compressive forces, when the AFR/PTFE surfaces are in contact, equal and opposite triboelectric charges, i.e. negative charges on PTFE and positive charges on AFR are generated. Upon the removal of the force, the presence of these opposite charges on the surfaces leads to the formation of the potential between the two metal electrodes, which can drive the electrons through an external circuit until the potential difference is neutralized. Thus, a higher charge density will produce a higher potential difference between the two TENG dielectric surfaces. It is clear that as compared to PA6, the significantly higher tribo-positive nature of the synthesized AFR layer can be further demonstrated by using SKPFM technique. 
The SKPFM measurements rely on the matching of tip bias $\mathrm{V}_{\mathrm{DC}}$ with the contact potential difference between the sample and the probe contact potential difference, $\mathrm{V}_{\mathrm{CPD}}$, by nullifying the vibration of the probe, which is initially driven by the electrostatic force, induced on the AFM probe. The relationship between the parameters and measurement conditions are detailed in the Supplementary Information. In the first instance, we have measured the surface potential of the as-prepared cured AFR sample (Fig. 6(a, b). For the pristine AFR sample, this average surface potential $\phi$ was measured to be $1.147 \mathrm{~V}$ which is significantly higher than that of the extensively used tribo-positive PA6, which showed a value of $0.875 \mathrm{~V}$ only [8]. A higher contact potential difference, $\mathrm{V}_{\mathrm{CPD}}$, implies that the electrons can be easily donated from the surface and the surface will gain a positive charge after contacting with the other materials. Thus, as compared to the PA6, a higher surface potential means that the AFR is more triboelectric positive, thus having a higher ability to donate electrons and explains the significantly higher performance for the AFR/PTFE TENG as compared to the PA6/PTFE TENG. It should also be noted that the average surface potential of AFR is quite close to our earlier reported value for polyethene oxide (1.160-1.266 V) which was shown to be a superior material as compared to PA6 [8]. The surface potential values for AFR are therefore in line with the earlier reports on the correlation between the surface functional groups and the ensuing surface charge on a material [15].

Besides the understanding of the surface potential of a triboelectric surface, it is equally important to understand the change in the surface potential and the spatial distribution of charges occurring upon the contact between the triboelectric materials. Fig. 6(a-h) showing the SKPFM plots of the topographic height and the surface potential of the AFR sample at various stages of contact with the charge injected PTFE by the ionization gun. It should be noted that the surface potential of the charge injected PTFE sample was too high, leading to channel saturation ( $\pm 10 \mathrm{~V}$ for our system) (see Fig. S5 and Fig. S6, Supplementary Information for the KPFM and electrostatic force microscopy (EFM) images) and even after multiple contact cycles remained too high to be measured, hence, the SKPFM discussion would be limited to the AFR samples alone. In addition, there is no cross-talk between the topographic features and surface potential was observed for the AFR samples.

By employing a method similar to that of Barnes et al. [43], the AFR and PTFE layers were repeatedly contacted/separated with each other for a number of cycles (TENG excited by hand, 1-2 Hz, 5-10 N) and then the surface potential was measured by using SKPFM. The delay between the contact and the completion of the scan was kept to a minimum so that the time- 
variant effect on surface potential could be minimized [43]. Due to the lack of metal contacts on the imaging surface (acting as reference points) and the relatively large area of the AFR samples, we could not ensure that the same area was being measured. However, repeated measurements have shown that the surface potential changes observed are consistent throughout the sample. The contact electrification between AFR and PTFE, after a nominal 30 contact cycles, leads to the exchange of charges between the AFR and PTFE and change the average CPD, $\mathrm{V}_{\mathrm{CPD}(\mathrm{av})}$ of AFR to $1.176 \mathrm{~V}$ (Fig 6(c, d)). As the cyclic contact increases to 60 cycles, $\mathrm{V}_{\mathrm{CPD}(\mathrm{av})}$ has a nominal increase to $1.187 \mathrm{~V}$ (Fig. 6(e, f)). This variation of nearly 40 $\mathrm{mV}$ is shown in Fig. 6(i), where the surface potential histogram shows a clear upshift of the AFR surface potential. This increase in the $\mathrm{V}_{\mathrm{CPD}(\mathrm{av})}$ can be considered to arise from a reduction in the overall roughness upon cycling resulting in better microscopic contact.

However, unlike the study by Baytekin et al. [44], for any of the contact cycled AFR surfaces, no mosaic charge distribution could be observed. However, when the AFR and PTFE surfaces were brought in contact for $10 \mathrm{sec}$, there was a significant shift in the surface potential for the AFR samples from 1.187 V to 1.206 V. However, any further cycling or contacting/rubbing of the AFR and PTFE surface did not affect the surface potential of the AFR visibly, and it remained around the $1.206 \mathrm{~V}$ value only. This trend of the upshift in the surface potential of the tribo-positive material is in line with that observed by Baytekin et al. and Barnes et al. [43, 44]. However, their use of soft, high tack PDMS ensures higher conformity of the tribo-surfaces leading to a significantly higher difference in the observed surface potentials (up to $100 \mathrm{mV}$ ) $[43,44]$. The surface charge density increase on the AFR surface by contact electrification can be calculated using the simple capacitor model [43]:

$$
\sigma=\frac{\varepsilon_{0} \Delta V_{C P D}}{h}
$$

where $\varepsilon_{0}$ is the vacuum permittivity $\left(8.854 \times 10^{-12} \mathrm{~F} / \mathrm{m}\right), \Delta V_{C P D}(+60 \mathrm{mV})$ is the $\mathrm{V}_{\mathrm{CPD}(\mathrm{av})}$ change of AFR before and after the contact with PTFE and $h$ is the lift height $(10 \mathrm{~nm})$ between the AFM tip and AFR film during the second pass of the SKPFM measurement. The calculated value of $53.12 \mu \mathrm{C} \mathrm{m}^{-2}$ matches quite well with the low-frequency low impact operation of the TENG, which showed a charge transfer value of nearly $53 \mu \mathrm{C} \mathrm{m}^{-2}$ (Fig. S3, Supplementary Information).

\section{Conclusions}

In summary, a facile approach for the synthesis of highly tribo-positive aniline formaldehyde 
resin is proposed. The ease of post-processing and curing steps, in conjunction with its suitable chemical functional groups, dielectric behaviour, and surface morphology makes AFR highly suitable for energy harvesting TENG applications. A thin $(\sim 117 \mu \mathrm{m})$ flat AFR dielectric film in an AFR/PTFE TENG gave a maximum peak-to-peak voltage of 1,000 V, a current density of $65 \mathrm{~mA} \mathrm{~m}^{-2}$ and a transferred charge density of $200 \mu \mathrm{C} \mathrm{m} \mathrm{m}^{-2}$, respectively. With each generation cycle, the AFR/PTFE TENG can harvest $\sim 28 \mu \mathrm{J}$ energy to a matched load resistor, which is twice the value of a PA6 tribo-positive layer based PA6/PTFE TENG. The improvement of the performance of the AFR/PTFE TENG is mainly attributed to the high surface potential of AFR (1.147 V) as confirmed by SKPFM. In addition, the AFR/PTFE TENG device was demonstrated to be able to light up 35 LEDs and have a highly stable output for a long test duration of over 16,000 cycles. The work, therefore, provides a simple, costeffective route for expanding the portfolio of tribo-positive materials for enabling highperformance TENG energy harvesters.

\section{Acknowledgement}

N. Soin gratefully acknowledges the partial financial support received from the National Research Foundation (NRF), South Africa (Grant No. ECR180426324617). Authors N. Soin, J. Luo and C. H. See would also like to acknowledge the support received from University of Bolton through the Jenkinson Award scheme. The authors would also like to thank Dr John Benson at the Ulster University for his help with some initial XPS measurements. XPS data collection was performed at the EPSRC National Facility for XPS ('HarwellXPS'), operated by Cardiff University and UCL, under contract No. PR16195.

\section{References}

[1] P.E. Shaw, Experiments on tribo-electricity. I.-The tribo-electric series, Proc R Soc Lond A. 94 (1917) 16-33. doi:10.1098/rspa.1917.0046.

[2] J. Henniker, Triboelectricity in Polymers, Nature. 196 (1962) 474. doi:10.1038/196474a0.

[3] H. Zou, Y. Zhang, L. Guo, P. Wang, X. He, G. Dai, H. Zheng, C. Chen, A.C. Wang, C. $\mathrm{Xu}$, Z.L. Wang, Quantifying the triboelectric series, Nat. Commun. 10 (2019) 1427. doi:10.1038/s41467-019-09461-X.

[4] F.-R. Fan, Z.-Q. Tian, Z. Lin Wang, Flexible triboelectric generator, Nano Energy. 1 (2012) 328-334. doi:10.1016/j.nanoen.2012.01.004.

[5] N. Soin, P. Zhao, K. Prashanthi, J. Chen, P. Ding, E. Zhou, T. Shah, S.C. Ray, C. Tsonos, T. Thundat, E. Siores, J. Luo, High performance triboelectric nanogenerators based on phase-inversion piezoelectric membranes of poly(vinylidene fluoride)-zinc stannate (PVDF-ZnSnO 3 ) and polyamide-6 (PA6), Nano Energy. 30 (2016) 470-480. 
doi:10.1016/j.nanoen.2016.10.040.

[6] P. Zhao, N. Soin, K. Prashanthi, J. Chen, S. Dong, E. Zhou, Z. Zhu, A.A. Narasimulu, C.D. Montemagno, L. Yu, J. Luo, Emulsion Electrospinning of Polytetrafluoroethylene (PTFE) Nanofibrous Membranes for High-Performance Triboelectric Nanogenerators, ACS Appl. Mater. Interfaces. 10 (2018) 5880-5891. doi:10.1021/acsami.7b18442.

[7] A.A. Narasimulu, P. Zhao, N. Soin, K. Prashanthi, P. Ding, J. Chen, S. Dong, L. Chen, E. Zhou, C.D. Montemagno, J. Luo, Significant triboelectric enhancement using interfacial piezoelectric $\mathrm{ZnO}$ nanosheet layer, Nano Energy. 40 (2017) 471-480. doi:10.1016/j.nanoen.2017.08.053.

[8] P. Ding, J. Chen, U. Farooq, P. Zhao, N. Soin, L. Yu, H. Jin, X. Wang, S. Dong, J. Luo, Realizing the potential of polyethylene oxide as new positive tribo-material: Over $40 \mathrm{~W} / \mathrm{m}$ 2 high power flat surface triboelectric nanogenerators, Nano Energy. 46 (2018) 63-72. doi:10.1016/j.nanoen.2018.01.034.

[9] H. Chen, Y. Xu, J. Zhang, W. Wu, G. Song, Enhanced stretchable graphene-based triboelectric nanogenerator via control of surface nanostructure, Nano Energy. 58 (2019) 304-311. doi:10.1016/j.nanoen.2019.01.029.

[10] Q. Ye, Y. Wu, Y. Qi, L. Shi, S. Huang, L. Zhang, M. Li, W. Li, X. Zeng, H. Wo, X. Wang, S. Dong, S. Ramakrishna, J. Luo, Effects of liquid metal particles on performance of triboelectric nanogenerator with electrospun polyacrylonitrile fiber films, Nano Energy. 61 (2019) 381-388. doi:10.1016/j.nanoen.2019.04.075.

[11] C. Lee, S. Yang, D. Choi, W. Kim, J. Kim, J. Hong, Chemically surface-engineered polydimethylsiloxane layer via plasma treatment for advancing textile-based triboelectric nanogenerators, Nano Energy. 57 (2019) 353-362. doi:10.1016/j.nanoen.2018.12.051.

[12] S.M. Harstad, P. Zhao, N. Soin, A.A. El-Gendy, S. Gupta, V.K. Pecharsky, J. Luo, R.L. Hadimani, Gd5Si4-PVDF nanocomposite films and their potential for triboelectric energy harvesting applications, AIP Adv. 9 (2019) 035116. doi:10.1063/1.5080116.

[13] Y. Xie, J. Long, P. Zhao, J. Chen, J. Luo, Z. Zhang, K. Li, Y. Han, X. Hao, Z. Qu, M. Lu, W. Yin, A self-powered radio frequency (RF) transmission system based on the combination of triboelectric nanogenerator (TENG) and piezoelectric element for disaster rescue/relief, Nano Energy. 54 (2018) 331-340. doi:10.1016/j.nanoen.2018.10.021.

[14] A. Coehn, Ueber ein Gesetz der Electricitätserregung, Ann. Phys. 300 (1898) 217-232. doi:10.1002/andp.18983000203.

[15] A.F. Diaz, R.M. Felix-Navarro, A semi-quantitative tribo-electric series for polymeric materials: the influence of chemical structure and properties, J. Electrost. 62 (2004) 277290. doi:10.1016/j.elstat.2004.05.005.

[16] Y.J. Kim, J. Lee, S. Park, C. Park, C. Park, H.-J. Choi, Effect of the relative permittivity of oxides on the performance of triboelectric nanogenerators, RSC Adv. 7 (2017) 4936849373. doi:10.1039/C7RA07274K.

[17] X.-S. Zhang, M.-D. Han, R.-X. Wang, B. Meng, F.-Y. Zhu, X.-M. Sun, W. Hu, W. Wang, Z.-H. Li, H.-X. Zhang, High-performance triboelectric nanogenerator with enhanced energy density based on single-step fluorocarbon plasma treatment, Nano Energy. 4 (2014) 123-131. doi:10.1016/j.nanoen.2013.12.016.

[18] J. Yang, J. Chen, Y. Liu, W. Yang, Y. Su, Z.L. Wang, Triboelectrification-Based Organic Film Nanogenerator for Acoustic Energy Harvesting and Self-Powered Active Acoustic Sensing, ACS Nano. 8 (2014) 2649-2657. doi:10.1021/nn4063616.

[19] Y. Xie, S. Wang, S. Niu, L. Lin, Q. Jing, J. Yang, Z. Wu, Z.L. Wang, Grating-structured freestanding triboelectric-layer nanogenerator for harvesting mechanical energy at $85 \%$ total conversion efficiency, Adv. Mater. Deerfield Beach Fla. 26 (2014) 6599-6607. doi:10.1002/adma.201402428.

[20] A. Gürses, Z. Eroğlu, K. Güneş, Ç. Doğar, Synthesis and Thermal and Textural 
Characterization of Aniline Formaldehyde-Organoclay Composites, Acta Phys. Pol. A. 129 (2016) 853-856. doi:10.12693/APhysPolA.129.853.

[21] G. Liu, M.S. Freund, New Approach for the Controlled Cross-Linking of Polyaniline: Synthesis and Characterization, Macromolecules. 30 (1997) 5660-5665. doi:10.1021/ma970469n.

[22] K. Kishore, K.N. Santhanalakshmi, A thermochemical approach to the analysis of anilineformaldehyde reactions, Thermochim. Acta. 64 (1983) 155-165. doi:10.1016/00406031(83)80139-7.

[23] J. Santhanalakshmi, Studies on the thermal decomposition of thermosetting anilineformaldehyde resins, Thermochim. Acta. 119 (1987) 321-327. doi:10.1016/00406031(87)80268-X.

[24] M.A. Quraishi, S.K. Shukla, Poly(aniline-formaldehyde): A new and effective corrosion inhibitor for mild steel in hydrochloric acid, Mater. Chem. Phys. 113 (2009) 685-689. doi:10.1016/j.matchemphys.2008.08.028.

[25] Y. Zhang, M. Nie, X. Wang, Y. Zhu, F. Shi, J. Yu, B. Hou, Effect of molecular structure of aniline-formaldehyde copolymers on corrosion inhibition of mild steel in hydrochloric acid solution, J. Hazard. Mater. 289 (2015) 130-139. doi:10.1016/j.jhazmat.2015.02.036.

[26] T. Maity, B.C. Samanta, S. Dalai, A.K. Banthia, Toughened epoxy with amine functional aniline formaldehyde condensate (AFAFC), Pigment Resin Technol. 35 (2006) 12-21. doi:10.1108/03699420610638003.

[27] F. Mustata, I. Bicu, Cyclohexanone-aniline-formaldehyde resins - synthesis and characterization, Polimery. (2002) 817-821.

[28] B.K. Kandola, J.R. Ebdon, C. Zhou, Development of vinyl ester resins with improved flame retardant properties for structural marine applications, React. Funct. Polym. 129 (2018) 111-122. doi:10.1016/j.reactfunctpolym.2017.08.006.

[29] J. Yamuna, T. Siva, S.S.S. Kumari, S. Sathiyanarayanan, A smart poly(anilineformaldehyde) microcapsule based self-healing anticorrosive coating, RSC Adv. 6 (2015) 79-86. doi:10.1039/C5RA19524A.

[30] K.-S. Ho, T.-H. Hsieh, C.-W. Kuo, S.-W. Lee, J.-J. Lin, Y.-J. Huang, Effect of aniline formaldehyde resin on the conjugation length and structure of doped polyaniline: Spectral studies, J. Polym. Sci. Part Polym. Chem. 43 (2005) 3116-3125. doi:10.1002/pola.20796.

[31] E. Ateş, N. Kızılcan, Aniline and oligoaniline modified cyclohexanone-formaldehyde resins, Pigment Resin Technol. 40 (2011) 29-35. doi:10.1108/03699421111095919.

[32] L. Kaßner, A. Knoblauch, A. Seifert, R.-E. Grützner, G. Cox, A. Lange, S. Csihony, F. Simon, S. Anders, L. Kroll, M. Rahaman, D. Zahn, L. Mertens, M. Weber, M. Mehring, S. Spange, Nanostructured Aniline Formaldehyde Resin/Polysilazane Hybrid Materials by Twin Polymerization, Macromol. Chem. Phys. 217 (2016) 2462-2472. doi:10.1002/macp.201600152.

[33] T.R. Gengenbach, R.C. Chatelier, H.J. Griesser, Correlation of the Nitrogen 1s and Oxygen 1s XPS Binding Energies with Compositional Changes During Oxidation of Ethylene Diamine Plasma Polymers, Surf. Interface Anal. 24 (1996) 611-619. doi:10.1002/(SICI)1096-9918(19960916)24:9<611::AID-SIA169>3.0.CO;2-7.

[34] M.E.V. Valkenburg, Reference Data for Engineers: Radio, Electronics, Computers and Communications, Elsevier, 2001.

[35] B.G. Soares, M.E. Leyva, G.M.O. Barra, D. Khastgir, Dielectric behavior of polyaniline synthesized by different techniques, Eur. Polym. J. 42 (2006) 676-686. doi:10.1016/j.eurpolymj.2005.08.013.

[36] S. Niu, S. Wang, L. Lin, Y. Liu, Y.S. Zhou, Y. Hu, Z.L. Wang, Theoretical study of contact-mode triboelectric nanogenerators as an effective power source, Energy Environ. Sci. 6 (2013) 3576. doi:10.1039/c3ee42571a. 
[37] Y. Zi, S. Niu, J. Wang, Z. Wen, W. Tang, Z.L. Wang, Standards and figure-of-merits for quantifying the performance of triboelectric nanogenerators, Nat. Commun. 6 (2015) 8376. doi:10.1038/ncomms9376.

[38] J. Wang, C. Wu, Y. Dai, Z. Zhao, A. Wang, T. Zhang, Z.L. Wang, Achieving ultrahigh triboelectric charge density for efficient energy harvesting, Nat. Commun. 8 (2017). doi:10.1038/s41467-017-00131-4.

[39] M. Taghavi, L. Beccai, A contact-key triboelectric nanogenerator: Theoretical and experimental study on motion speed influence, Nano Energy. 18 (2015) 283-292. doi:10.1016/j.nanoen.2015.10.019.

[40] J. Chen, W. Xuan, P. Zhao, U. Farooq, P. Ding, W. Yin, H. Jin, X. Wang, Y. Fu, S. Dong, J. Luo, Triboelectric effect based instantaneous self-powered wireless sensing with selfdetermined identity, Nano Energy. 51 (2018) 1-9. doi:10.1016/j.nanoen.2018.06.029.

[41] A.M. Barnes, A.D. Dinsmore, Heterogeneity of surface potential in contact electrification under ambient conditions: A comparison of pre- and post-contact states, J. Electrost. 81 (2016) 76-81. doi:10.1016/j.elstat.2016.04.002.

[42] H.T. Baytekin, A.Z. Patashinski, M. Branicki, B. Baytekin, S. Soh, B.A. Grzybowski, The Mosaic of Surface Charge in Contact Electrification, Science. 333 (2011) 308-312. doi:10.1126/science.1201512. 